\title{
Peroxynitrite induced fibrinogen site identification
}

\author{
Yunjing Luo*, Jianlong Shi and Jingjing Li \\ College of Life Science and Bioengineering, Beijing University of Technology, 100124 P.R. China
}

\begin{abstract}
An increasing number of peroxynitrite-mediated fibrinogen nitrifications have been associated with thrombotic diseases. However, few reports related to priority nitrified fibrinogen injury sites exist. In this paper, an improved method, which simulated the environment in vivo, was used to inspect the structural changes of fibrinogen treated with peroxynitrite and LC-MS/MS in order to investigate the fibrinogen injury sites. The SDS-PAGE results indicated that $\gamma$ subunits of Fg were vulnerable to oxidative/nitrative modifications induced by peroxynitrite. An in-depth analysis of fibrinogen $\gamma$ chain (Fg I) nitration site identification and susceptibility to peroxynitrite-utilizing LC-MS/MS strategy was performed. Based on a large dataset, the results indicated the priority injury sites during database searching were Y96, Y262, Y274, Y348, and Y363; these results could be applied to biomedical studies.
\end{abstract}

Keywords: Fibrinogen, LC-MS/MS, SDS-PAGE, peroxynitrite, tyrosine nitration

\section{Introduction}

Fibrinogen $(\mathrm{Fg})$ is a $340 \mathrm{kDa}$ plasma protein, which is converted into fibrins by thrombin during blood coagulation. It consists of two sets of three polypeptide chains, termed $\mathrm{A} \alpha, \mathrm{B} \beta$, and $\gamma$, and its dimer is joined by disulfide bonds to form a central hydrophobic N-terminal $\mathrm{E}$ domain connected to two outer hydrophobic C-terminal D domains [1], as shown in Figure 1 [2]. Each D domain is connected to the E domain through a flexible coiled-coil section, allowing the protein to bend. $\mathrm{Fg}$ structural changes significantly impact biological activity, thereby playing an essential role in some cardiovascular diseases.

The toxicant Peroxynitrite (PN), derived from a diffusion-controlled interaction between $\mathrm{O}_{2}{ }^{-}{ }^{-}$and $\mathrm{NO}$, can damage a wide array of plasma albumins in vivo, leading to serious biological consequences [3]. Nitrotyrosine is recognized as an indicator of oxidative injury and inflammation in proteins [4]. As a result of this post-translational modification induced by $\mathrm{ONOO}^{-}$, protein spatial conformation and associated functions are usually altered [5]. In several cases, the identification of specific nitrated proteins has assisted in illuminating the molecular mechanisms of diseases, thus inducing atherosclerosis disorders [6] and coronary artery disease [7].

The effect of nitrification on fibrinogen function has been intensely investigated. Many researchers have concluded that $\mathrm{ONOO}^{-}$would destroy and inactivate fibrinogen [8]. The identification of predominantly nitrated fibrinogen tyrosine sites could contribute to targeted drug development.

\footnotetext{
*Address for correspondence: Yunjing Luo, College of Life Science and Bioengineering, Beijing University of Technology, 100 Ping Le Yuan, Chaoyang District, Beijing 100124, China. Tel.:+86-10-6739-1667; Fax: +86-10-67391667; E-mail: luoyj@bjut.edu.cn.
} 
However, since a 70 tyrosine residues are involved in the three fibrinogen subunits, the determination of sites of interest remains enigmatic. In this study, the LC-MS/MS method was successfully utilized to confirm the insulin [9] and p16 protein [10] nitration sites induced by $\mathrm{ONOO}^{-}$. Tang et al. [11] investigated the tryptic digests of nitrated fibrinogen in human biofluids; although 26 different nitrated tyrosine sites were detected, the Tyr-containing sequence coverage was not high enough to identify the sites that were more likely to suffer injury. Since fibrinogen $\gamma$ chains (Fg I) are most susceptible, they could be a signature for several oxidative stress pathologies.

Considering this information, the selectivity and priority of Fg I nitration sites were characterized using high-resolution liquid chromatography coupled with tandem mass spectrometry analysis, and the MS/MS spectra were compared to the MASCOT database in order to assist in drug design for related diseases.

\section{Materials and methods}

\subsection{Materials}

Bovine fibrinogen was purchased from Sigma-Aldrich (USA). Fibrinogen fraction I ( $\gamma$ chains) was obtained from TCI (Shanghai). The protein molecular weight marker for electrophoresis was obtained from Shenzheng Biotechnology (Shanghai). Dithiothreitol and iodoacetamide were obtained from Merck (Darmstadt, Germany). Peroxynitrite was synthesized according to the Uppu et al. operation approach [12]. The prepared stock solution was aliquoted in $0.1 \mathrm{M} \mathrm{NaOH}$, and its concentration was determined by spectrophotometry $\left(\varepsilon_{302}=1670 \mathrm{M}^{-1} \cdot \mathrm{cm}^{-1}\right)$ immediately before use. All other chemicals were analytical-grade and commercially pure.

\subsection{SDS-PAGE analysis}

Polyacrylamide gel electrophoresis (12\%) in the presence of sodium dodecyl sulfate (SDS) was performed according to the Tetik procedure [13]. Bovine fibrinogen $(1 \mathrm{mg} / \mathrm{mL})$ in PBS ( $\mathrm{pH} 7.4)$ was induced by sequential titration with the obtained peroxynitrite to different final concentrations $(0 \mathrm{mM}$, $0.5 \mathrm{mM}, 1 \mathrm{mM}, 1.5 \mathrm{mM}, 2 \mathrm{mM}$, and $2.5 \mathrm{mM}$ ) and incubated for $30 \mathrm{~min}$ at $37^{\circ} \mathrm{C}$. The protein gels were stained with Coomassie brilliant blue (R250).

\subsection{Preparation of nitrated Fg I and enzymatic digests}

Bovine fibrinogen $\gamma$ chains (Fg I, $1 \mathrm{mg} / \mathrm{mL}$ ) in PBS (pH 7.4) were induced by the sequential titration of the obtained peroxynitrite to different final concentrations $(0 \mathrm{nM}, 0.3 \mathrm{nM}, 1 \mathrm{nM}, 3.3 \mathrm{nM}$, $10 \mathrm{nM}$ ) with a water bath for $30 \mathrm{~min}$ at $37^{\circ} \mathrm{C}$. Then, the protein samples were denatured with $50 \mathrm{mM}$ Tris( $\beta$-chloroethyl) phosphate(TCEP) in a $25 \mathrm{mM} \mathrm{NH}_{4} \mathrm{HCO}_{3}$ buffer $(\mathrm{pH} \mathrm{8.0)}$ and incubated for $1 \mathrm{~h}$ at $56^{\circ} \mathrm{C}$. The reduced peptides were alkylated with iodoacetamide to a $50 \mathrm{mM}$ concentration for $1 \mathrm{~h}$ at room temperature in the dark. Subsequently, the fibrinogens were digested with trypsin/Glu-C (chymotrypsin was added to the native $\mathrm{Fg} \mathrm{I}$ ) at a protein ratio of $1: 50 \mathrm{mixture}(\mathrm{w} / \mathrm{w})$ overnight at $37^{\circ} \mathrm{C}$ with gentle shaking. The resulting peptides were desalted with $0.1 \%$ trifluoroacetic acid (TFA) $/ 2 \%$ acetonitrile solution prior to lyophilization, then vacuum centrifuged at $20000 \mathrm{~g}$ for $30 \mathrm{~min}$. The supernatant fractions were collected for peptide sequencing. 


\subsection{Identification of Fg I nitration sites with LC-MS/MS}

The site identification was performed using a high-performance capillary LC system (NanoLC-2D, Eksigent Technologies, CA) coupled online with the LTQ ion trap mass spectrometer (Finnigan, Thermo, San Jose, CA) via an in-house prepared needle tip and nanospray source. The column was packed with $5 \mu \mathrm{m}$ particles with $300 \AA$ diameter pores (PepMap 300, Dionex, USA) into a $75 \mu \mathrm{m} \times 15$ $\mathrm{cm} \mathrm{C18-bonded} \mathrm{silica} \mathrm{capillary.} \mathrm{The} \mathrm{mobile} \mathrm{phases} \mathrm{consisted} \mathrm{of} \mathrm{A} \mathrm{(} 2 \%$ acetonitrile, $0.1 \%$ TFA, $98 \%$ water) and B (20\% water, $0.1 \%$ TFA, $80 \%$ acetonitrile). The peptide separation was conducted as a linear gradient using the following program: from $2 \%$ to $40 \%$ B over $90 \mathrm{~min}$ and to $100 \%$ B in $15 \mathrm{~min}$, and lasted it for the same while, finally eluted at $100 \%$ A for $30 \mathrm{~min}$ at a flow rate of $0.3 \mu \mathrm{L} / \mathrm{min}$. Peptide ions were acquired in a survey scan across the $\mathrm{m} / \mathrm{z}$ range $(350-1600)$ followed by the data-dependent MS/MS mode ( $35 \%$ normalized collision energy). The ion spray voltage was operated at $2.2 \mathrm{kV}$. The data was collected for $130 \mathrm{~min}$ at $200^{\circ} \mathrm{C}$.

\subsection{Data processing}

The MS/MS spectra were compared to the MASCOT (version 2.1.0) database [14] which contains all the sequences of fibrinogen subunits. In the presence of peroxynitrite, the following criteria were used for further positioning of the targeted nitrotyrosines: variable modification of methionine $(+15.99$ Da) corresponding to single oxygen, nitration modification $(+44.9851 \mathrm{Da})$ matching the mono-nitro on tyrosine residues, and an additional mass of $90 \mathrm{Da}$ or $135 \mathrm{Da}$ corresponding to the di-nitro or tri-nitrotyrosine species. The primary error and secondary error were $1.5 \mathrm{Da}$ and $0.8 \mathrm{Da}$, respectively. The peptide identifications were analyzed as previously described [15].

\section{Results}

\subsection{Analysis of native and nitrated fibrinogen using SDS-PAGE}

The fibrinogen exposed to peroxynitrite $(0.5-2.5 \mathrm{mM})$ changed distinctly in its electrophoretic running gel (Figure 2). Not only were the bands of $\mathrm{A} \alpha(66 \mathrm{KDa}), \mathrm{B} \beta(52 \mathrm{KDa})$, and $\gamma(46 \mathrm{KDa})$ chains observed, but also some of the new high molecular weight (HMW) bands on the top. As the concentration of peroxynitrite increased, the $\gamma$ chain weakened and disappeared, followed by the B $\beta$ chains, indicating that the three fibrinogen polypeptide chains were injured in the order of $\gamma, \mathrm{B} \beta$, and $\mathrm{A} \alpha$. Furthermore, the HMW aggregate could be associated with the dityrosine crosslink formation between A $\alpha$ chains [16].

\subsection{Analysis of native and nitrated fibrinogen $\gamma$ chains using precursor-ion scanning (LC-MS/MS)}

Trypsin, chymotrypsin, and Glu-C were employed and supplemented during digestion in order to ensure the accuracy of the detection results. The high coverage of the MS/MS spectra easily matched to correct the peptides even though the tryptic peptides existed up to two non-cleavage sites. The sequence coverage was $95.6 \%$ and $97.6 \%$ before and after the protein nitration, respectively. Hence, the total peptide sequence amount was large enough to expediently evaluate the $100 \%$ tyrosine coverage.

Fibrinogen $\gamma$ chains (Fg I) were digested into peptides by trypsin under the action of $\mathrm{ONOO}^{-}$. The resulting peptides were separated using liquid chromatography (LC) and tandem electrospray mass 
spectrometry with automatic fraction collection. Each fraction was subsequently searched for and analyzed in the database as described previously. As an example, the LC-MS/MS analysis of Fg I nitrated with $\mathrm{ONOO}^{-}$at a $1 \mathrm{nM}$ solution concentration is shown in Figure 3. Figure 3a presents the total-ion current chromatographic traces collected during LC over a large time scale. The base peak chromatogram was similar to the UV chromatogram $(215 \mathrm{~nm})$. The corresponding mass spectra yielded a molecular weight of the precursor ions triggered by the survey scan. Figures $3 \mathrm{~b}$ and $3 \mathrm{c}$ show a single MS/tandom MS spectra at the $31.5 \mathrm{~min}$ and $\mathrm{m} / \mathrm{z} 814.6$ analysis point. Numerous peptide ion signals were observed in the $\mathrm{m} / \mathrm{z}$ scan data. The precursor-ions of interest for the MS/MS spectrum were extracted in order to acquire mass spectral information on the molecular weight and heterogeneity of the modified proteins.

After acquisition of the MS/MS spectra, a list of possible peptide molecular weights was obtained. The best-matching peptides suffered a rigorous algorithm. The nitration extent was evaluated by integrating the repetitive identification of the same peptide in one or multiple fractions, which were consistent in their theoretical predictions. This dataset provided a unique pointcut that indicated the location of nitrotyrosine sites. The representative data are discussed below. As suggested in Table 1, among the 20 observed tyrosine residues, not only the amount but also the nitration extent of the identified nitrotyrosine sites decreased as the $\mathrm{ONOO}^{-}$solution concentration decreased. When exposed to $3.3 \mathrm{nM} \mathrm{ONOO}-, 15$ species with mostly di-nitro and tri-nitro tyrosine formed; however, when exposed to the $1 \mathrm{nM}$ inducer, only 9 fewer-nitro tyrosine sites formed. After Fg I was treated with a trace amount of $\mathrm{ONOO}^{-}(0.3 \mathrm{nM})$, three mono-nitro identified sites and two di-nitro identified sites formed. Furthermore, the nitration sensitivity was significantly demonstrated. Y96, Y262, Y274, Y348, and Y363 were corroborated to be five susceptible nitration targets; their locations in the fibrinogen molecule are shown in Figure 4 [17].

In addition, the precursor ion scanning MS/MS of a tryptic digest peptide was compared: 89-108 in the absence and presence of $\mathrm{ONOO}^{-}$, as displayed in Figures 5a and 5b, respectively. A database search for tyrosine-containing peptides, with a shift of $135 \mathrm{Da}$ in the mass (from 1474.7 Da to 1609.7 $\mathrm{Da}$ ), suggested that three nitro groups were added, which provided adequate evidence for the Y96 identification of tri-nitrated species. In addition, Y96 required a molarity relatively low to $\mathrm{ONOO}^{-}$ modification and was a priority nitration site.

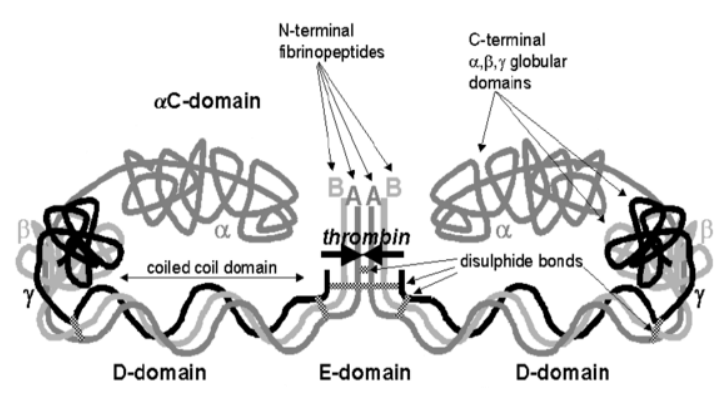

Fig. 1. The structure of fibrinogen.

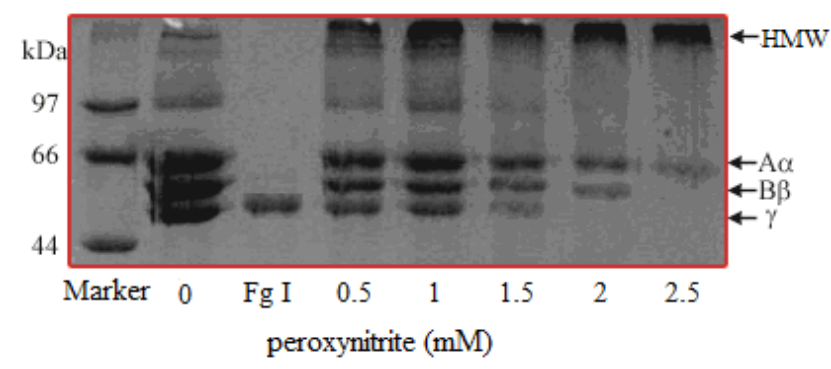

Fig. 2. SDS-PAGE of native and oxidized fibrinogen induced by different peroxynitrite concentrations. 

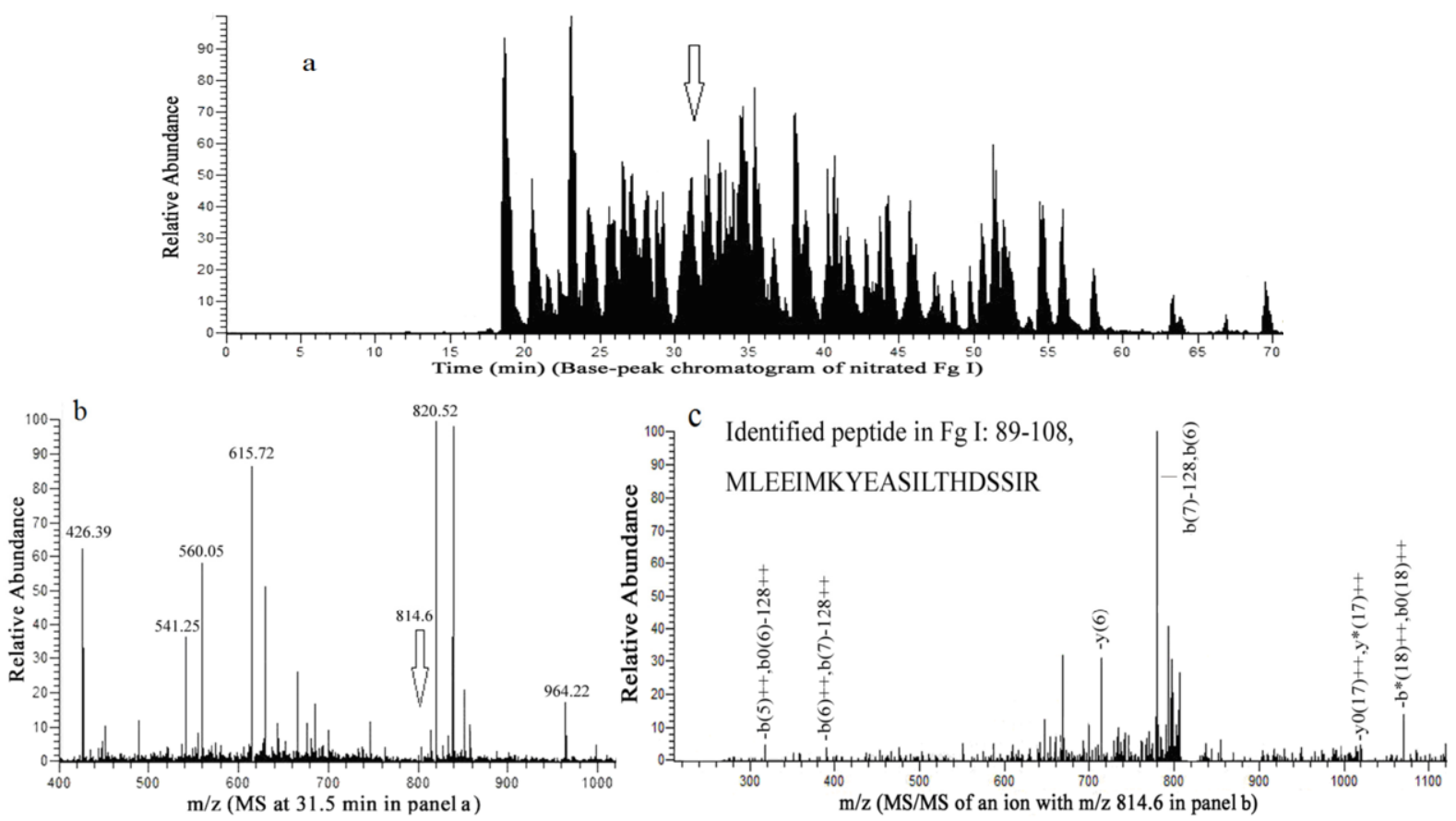

Fig. 3. LC-MS/MS analysis of digested fibrinogen $\gamma$ chains (Fg I) nitrated with $\mathrm{ONOO}^{-}$(1 $\mathrm{nM}$ solution concentration). (a) Total ion chromatograms of peroxynitrite-induced Fg I. Peptides were selected for MS/MS analysis. (b) The MS survey scan time was $31.5 \mathrm{~min}$. The co-eluting peptide ions were sequentially selected for further sequence identification. (c) MS/MS spectra of precursor ion with $814.6 \mathrm{~m} / \mathrm{z}$. Some b- and y-ions indicated on the spectra were derived from the peptide ion.

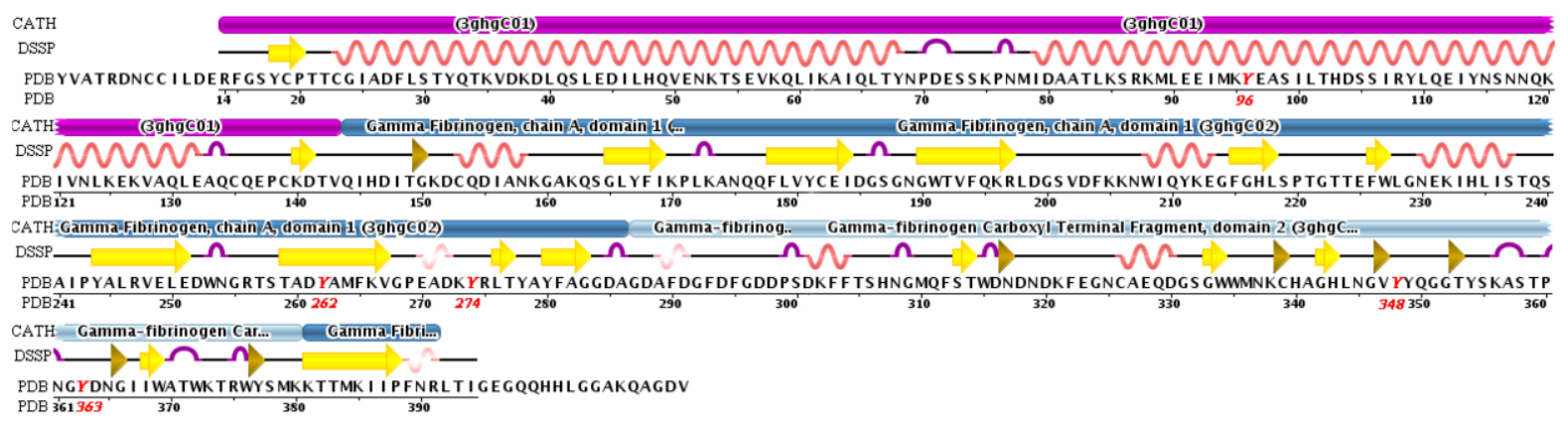

Fig. 4. Primary structure of fibrinogen I with nitration sites (in red and italics) (PDB entry 3ghg). 

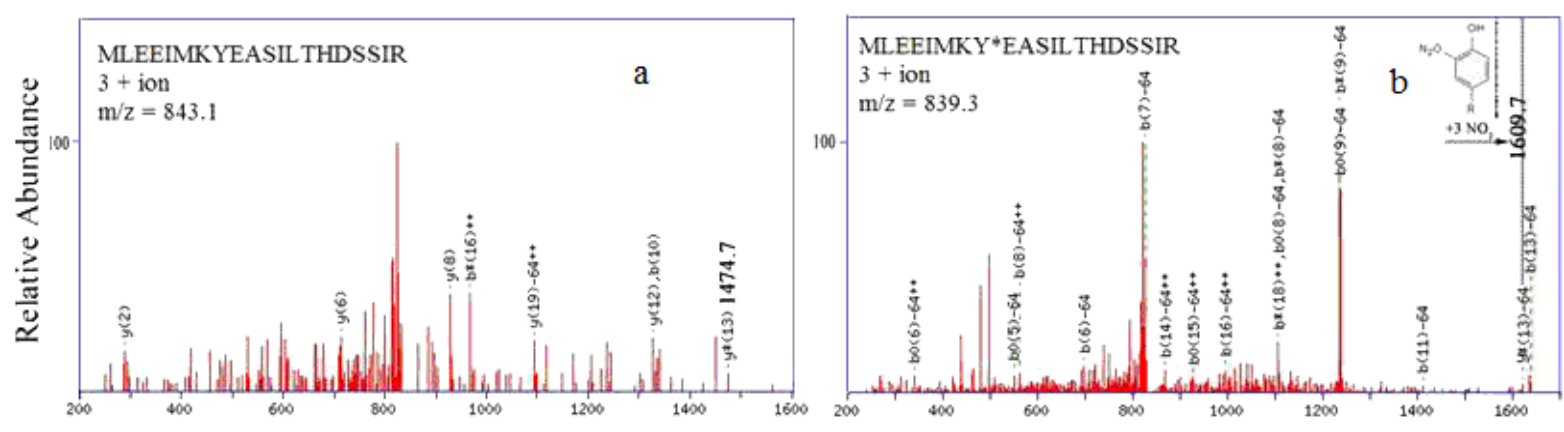

$\mathrm{m} / \mathrm{z}$ (Daltons/Unit of Charge)

Fig. 5. MS/MS Fragmentation peaks of peptide, 89-108: MLEEIMKYEASILTHDSSIR from the tryptic digestion of fibrinogen $\gamma$ chains. (a): native peptide, (b): nitrated peptides (final concentration of $\mathrm{ONOO}^{-}$was $3.3 \mathrm{nM}$ ).

Table 1

LC-MS/MS data of identified in vitro-nitrated fibrinogen peptides in different $\mathrm{ONOO}^{-}$final concentrations

\begin{tabular}{|c|c|c|c|c|c|}
\hline $\begin{array}{l}\text { Final } \\
\text { concentra } \\
\text { tion of } \\
\mathrm{ONOO}^{-} \\
\end{array}$ & $\begin{array}{l}\text { Nitrated Tyr } \\
\text { coverage } \\
{[\mathrm{NY} / \mathrm{Y}(\%} \\
\text { nitration })]\end{array}$ & $\begin{array}{l}\text { Mr. } \\
(\text { expt) }\end{array}$ & $\begin{array}{l}\text { Mr. } \\
\text { (calc) }\end{array}$ & Identified peptide sequence & Tyrosine site \\
\hline \multirow[t]{9}{*}{$3.3 \mathrm{nM}$} & \multirow[t]{9}{*}{$75 \%(15 / 20)$} & 1917.7 & 1918.8 & $1-14:$ YVATRDNCCILDER.F & Y1:Nitro3Y (Y) \\
\hline & & 2937.2 & 2938.2 & 15-38:R.FGSYCPTTCGIADFLSTYQTKVDK.D & $\begin{array}{l}\text { Y18: Nitro3Y (Y) } \\
\text { Y32: Nitro (Y) }\end{array}$ \\
\hline & & 4252.8 & 4251.8 & $\begin{array}{l}89-120: \text { K.MLEEIMKYEASILTHDSS } \\
\text { IRYLQEIYNSNNQK.I }\end{array}$ & $\begin{array}{l}\text { Y96*: Nitro3Y (Y) } \\
\text { Y109: Nitro3Y (Y) } \\
\text { Y114: Nitro (Y) }\end{array}$ \\
\hline & & 1594.7 & 1593.9 & 160 - 173:K.GAKQSGLYFIKPLK.A & Y167: Nitro (Y) \\
\hline & & 3302.6 & 3301.5 & $\begin{array}{l}206 \text {-232:K.KNWIQYKEGFGHLSPT } \\
\text { GTTEFWLGNEK.I }\end{array}$ & Y211: Nitro3Y (Y) \\
\hline & & 2390.5 & 2389.9 & 257-275:R.TSTADYAMFKVGPEADKYR.L & $\begin{array}{l}\text { Y262*: Nitro2Y (Y) } \\
\text { Y274*: Nitro3Y (Y) }\end{array}$ \\
\hline & & 3103.8 & 3103.1 & $\begin{array}{l}\text { 276-302:R.LTYAYFAGGDAGDAFD } \\
\text { GFDFGDDPSDK.F }\end{array}$ & $\begin{array}{l}\text { Y278: Nitro3Y (Y) } \\
\text { Y280: Nitro3Y (Y) }\end{array}$ \\
\hline & & 2102.2 & 2100.9 & $\begin{array}{l}339-356: \text { K.CHAGHLNGVYYQGG } \\
\text { TYSK.A }\end{array}$ & Y348*: Nitro2Y (Y) \\
\hline & & 3071.4 & 3070.3 & $\begin{array}{l}\text { 357 - 380:K.ASTPNGYDNGIIWAT } \\
\text { WKTRWYSMK.K }\end{array}$ & $\begin{array}{l}\text { Y363*: Nitro3Y (Y) } \\
\text { Y377: Nitro2Y (Y) }\end{array}$ \\
\hline \multirow[t]{6}{*}{$1 \mathrm{nM}$} & \multirow[t]{6}{*}{$45 \%(9 / 20)$} & 4027.9 & 4026.8 & $\begin{array}{l}89-120: K . M L E E I M K Y E A S I L T H D S S \\
\text { IRYLQEIYNSNNQK.I }\end{array}$ & $\begin{array}{l}\text { Y96*: Nitro (Y) } \\
\text { Y109: Nitro2Y (Y) }\end{array}$ \\
\hline & & 3302.7 & 3301.5 & $\begin{array}{l}206-232: K . K N W I Q Y K E G F G H L S P T \\
\text { GTTEFWLGNEK.I }\end{array}$ & Y211: Nitro3Y (Y) \\
\hline & & 2390.3 & 2389.9 & 257 - 275: R.TSTADYAMFKVGPEADKYR.L & $\begin{array}{l}\text { Y262*: Nitro2Y (Y) } \\
\text { Y274*: Nitro3Y (Y) }\end{array}$ \\
\hline & & 2924.6 & 2923.1 & $\begin{array}{l}276-302 \text { :R.LTYAYFAGGDAGDAF } \\
\text { DGFDFGDDPSDK.F }\end{array}$ & Y280: Nitro2Y (Y) \\
\hline & & 2056.8 & 2055.9 & 339 - 356: K.CHAGHLNGVYYQGGTYSK.A & Y348*: Nitro (Y) \\
\hline & & 2980.8 & 2980.3 & $\begin{array}{l}357 \text { - 380:K.ASTPNGYDNGIIWAT } \\
\text { WKTRWYSMK.K }\end{array}$ & $\begin{array}{l}\text { Y363*: Nitro2Y(Y) } \\
\text { Y377: Nitro }(Y)\end{array}$ \\
\hline $0.3 \mathrm{nM}$ & $25 \%(5 / 20)$ & 3937.7 & 3936.8 & 89-120:K.MLEEIMKYEASILTHDSS & Y96*: Nitro (Y) \\
\hline
\end{tabular}




\begin{tabular}{|l|l|l|l|l|}
\hline & & & IRYLQEIYNSNNQK.I & \\
\cline { 3 - 6 } & 2344.3 & 2344.9 & $257-275:$ R.TSTADYAMFKVGPEADKYR.L & $\begin{array}{l}\text { Y262*: Nitro2Y (Y) } \\
\text { Y274*: Nitro2Y (Y) }\end{array}$ \\
\cline { 3 - 6 } & 2054.5 & 2055.9 & $339-356:$ K.CHAGHLNGVYYQGGTYSK.A & Y348*: Nitro (Y) \\
\cline { 2 - 6 } & 2890.1 & 2890.3 & $\begin{array}{l}357-380: \text { Y.ASTPNGYDNGIIWATW } \\
\text { KTRWYSMK.K }\end{array}$ & Y363*: Nitro (Y) \\
\hline
\end{tabular}

Note: The position of fibrinogen nitrotyrosines (denoted in bold font) in the primary sequence is indicated by their corresponding numbers. [NY/Y (\%)], an estimate of the extent of nitration, is based on the number of nitrated peptides on the observed spectra. Tyrosine sites designated by asterisks were vulnerable to nitration. Mr indicates the molecular weight (Da) of the corresponding peptides. "expt" and "calc" are abbreviations of experiment and calculation, respectively.

\section{Discussion and conclusion}

Since fibrinogen (Fg) injury induced by $\mathrm{ONOO}^{-}$is closely associated with cardiovascular diseases [18], the nitration sites and extents of fibrinogen were identified using LC-MS/MS. As suggested from the SDS-PAGE gel, fibrinogen polypeptide chains were damaged in the order of $\gamma, \mathrm{B} \beta$, and $\mathrm{A} \alpha$ as the $\mathrm{ONOO}^{-}$amount increased. The $\gamma$ chains broke apart and disappeared when the final $\mathrm{ONOO}^{-}$ concentration reached $1.5 \mathrm{mM}$.

The fibrinogen $\gamma$ chains were subjected to a series of limited proteolysis and MS analyses in order to identify the preferential nitration sites. Molecular weight measurements could reveal a relatively detailed picture of nitrotyrosine sites by automatically searching the mass spectral data for characteristic $\mathrm{m} / \mathrm{z}$ differences. Considering the $\mathrm{ONOO}^{-}$levels in a physiological setting, the potential final concentration of $\mathrm{ONOO}^{-}$was employed in vivo. The results indicated that the decrease in the amount of nitro added to tyrosine sites was nearly proportional to the decline of the identified sites over incubation time. Even the lowest levels of peroxynitrite $(0.3 \mathrm{nM})$ could cause the cleavage and modification of fibrinogen. Five nitrotyrosines were identified as highly susceptible to nitration attack. This occurrence is completely probably in pathological conditions. The protein cytotoxicity and injury caused by $\mathrm{ONOO}^{-}$was demonstrated.

Among the five tyrosine sites detected for primarily nitration, Y262, Y274, Y348, and Y363 were located in the domain 2: C-terminal fragment; another site, Y96, was present in the $\mathrm{N}$-terminal within the fibrinogen molecule; and domain 1 (chain A) did not contain the susceptible nitration targets. As indicated by comparing the structural partition of the $\gamma$ chain, the $\mathrm{C}$ domains primarily contact the aqueous solution and undergo the majority of the modifications. The accessible surface around these nitrated tyrosine sites were characteristic in loops, and thereby crucial to the physiological activity of fibrinogen.

Determining the locations and heterogeneity of tyrosine in fibrinogen is valuable to oxidative stress-induced pathological studies. Even though it is only a beginning to structural characterization, the sensitivity of tyrosine sites to peroxynitrite could be as a useful reference in cardiovascular disease drug design.

\section{Acknowledgments}

This work was supported by the Beijing Natural Science Foundation (No. 2102005), the General program of science and technology development project of the Beijing Municipal Education Commission (KM201210005032), and the National Natural Science Foundation of China (No. 
20875006).

\section{References}

[1] Matsuda, Structure and function of fibrinogen inferred from hereditary dysfibrinogens, Fibrinolysis and Proteolysis $\mathbf{1 4}$ (2000), 187-197.

[2] S. Cavalu, V. Simon, C. Deleanu, et al., Fibrinogen adsorption onto bioglass aluminosilicates, Romanian Journal of Biophysics 17 (2007), 237-245.

[3] H. Ischiropoulos and A.B. Al-Mehdi, Peroxynitrite-mediated oxidative protein modifications, FEBS Letters 364 (1995), 279-282.

[4] Y Ding, Y.J. Luo and J. Fu, Effects of Mn (II) on peroxynitrite nitrifying fibrinogen, Bio-Medical Materials and Engineering 24 (2014), 901-907.

[5] P. Nowak, H.M. Zbikowska, M. Ponczek, et al., Different vulnerability of fibrinogen subunits to oxidative/nitrative modifications induced by peroxynitrite: Functional consequences, Thrombosis Research 121 (2007), 163-174.

[6] L.D. Buttery, D.R. Springall, A.H. Chester, et al., Inducible nitric oxide synthase is present within human atherosclerotic lesions and promotes the formation and activity of peroxynitrite, Laboratory Investigation 75 (1996), $77-85$.

[7] C. Vadseth, J.M. Souza, L. Thomson, et al., Pro-thrombotic state induced by post-translational modification of fibrinogen by reactive nitrogen species, The Journal of Biological Chemistry 279 (2004), 8820-8826.

[8] G. Lupidi, M. Angeletti, A.M. Eleuteri, et al., Peroxynitrite-mediated oxidation of fibrinogen inhibits clot formation, FEBS Letters 462 (1999), 236-240.

[9] Y. Wang, Y. Luo, R. Zhong, et al., Identification of sites of insulin nitration by peroxynitrite and characterization of its structural change, Protein Peptide Letters 15 (2008), 1063-1067.

[10] Y.J. Luo, J.J. Li, N. Zhang, et al., Identification of nitration sites by peroxynitrite on p16 protein, The Protein Journal 31 (2012), 393-400.

[11] Z.W. Tang, H. Wu, D. Du, et al., Sensitive immunoassays of nitrated fibrinogen in human biofluids, Talanta 81 (2010), $1662-1669$.

[12] R.M. Uppu and W.A. Pryor, Synthesis of peroxynitrite in a two-phase system using isoamyl nitrite and hydrogen peroxide, Analytical Biochemistry 236 (1996), 242-249.

[13] S. Tetik, E. Eksioglu-Demiralp and K.T. Yardimci, Effect of imatinib mesylate on platelet aggregation and fibrinogen binding to isolated platelets, Advances in Molecular Medicine 1 (2005), 165-170.

[14] Matrixscience, Available at: www.matrixscience.com/, last visit date: 2013-11-23.

[15] C.A. Sacksteder, W.J. Qian, T.V. Knyushko, et al., Endogenously nitrated proteins in mouse brain: Links to neurodegenerative disease, Biochemistry 45 (2006), 8009-8022.

[16] B. Lipinski, Pathophysiology of oxidative stress in diabetes mellitus, Journal of Diabetes and Its Complications $\mathbf{1 5}$ (2001), 203-210.

[17] Protein Data Bank (PDB entry 3ghg), Available at://www.rcsb.org, last visit date: 2015-01-07.

[18] P. Nowak, H.M. Zbikowska, M. Ponczek, et al., Different vulnerability of fibrinogen subunits to oxidative/nitrative modifications induced by peroxynitrite: Functional consequences, Thrombosis Research 121 (2007), 163-174. 